\title{
BMJ Open Evaluation of the efficacy and safety of a Chinese herbal formula (RCM-106) for atopic dermatitis: study protocol for a randomised, double-blind, placebo-controlled trial in children
}

\author{
Hsiewe Ying Tan, ${ }^{1}$ Anthony L Zhang, ${ }^{1}$ Charlie C Xue, ${ }^{1,2}$ Dacan Chen, ${ }^{2}$ \\ Cliff Da Costa, ${ }^{3}$ George B Lenon ${ }^{1}$
}

To cite: Tan HY, Zhang AL, Xue CC, et al. Evaluation of the efficacy and safety of a Chinese herbal formula (RCM-106) for atopic dermatitis: study protocol for a randomised, doubleblind, placebo-controlled trial in children. BMJ Open 2013;3:e003906.

doi:10.1136/bmjopen-2013003906

- Prepublication history for this paper is available online. To view these files please visit the journal online (http://dx.doi.org/10.1136/ bmjopen-2013-003906).

Received 28 August 2013 Revised 12 November 2013 Accepted 13 November 2013

CrossMark

For numbered affiliations see end of article.

Correspondence to Dr George Binh Lenon; george.lenon@rmit.edu.au

\section{ABSTRACT}

Introduction: Atopic dermatitis is a chronic, inflammatory skin rash that greatly affects quality of life. The current therapies are inadequate in managing atopic dermatitis and often have associated adverse effects or drug tolerance development. Chinese medicine is expected to have promising prospects in the management of atopic dermatitis and recent studies have shown encouraging results. This study aims to evaluate the efficacy and safety of a newly formulated Chinese herbal formula, RMIT Chinese Medicine-106 (RCM-106), in the management of moderate-to-severe atopic dermatitis in children aged 6-18 years.

Methods: The study is a randomised, double-blind, placebo-controlled, parallel-armed clinical trial. Participant, investigator and assessors will remain blinded to the treatment assignment until after the study has been completed. After a 2-week run-in period, 90 participants will be randomised, using block randomised sequences generated by computer, to receive either RCM-106 or matching placebo capsules, twice daily, for a treatment period of 8 weeks and followed up for 4 weeks. Primary outcome measures include the evaluation of disease severity and extent using two validated scoring instruments-Scoring Atopic Dermatitis (SCORAD) and Patient-Oriented Scoring Atopic Dermatitis (PO-SCORAD). Secondary outcome measures include the evaluation of quality of life using the Children's Dermatology Life Quality Index (CDLQI); occurrence of adverse events and total usage of other therapies as recorded in the participants' daily diary and laboratory studies which include eosinophil count, total IgE, full blood count and liver and kidney function tests. Intention-to-treat analysis will be applied to all data analyses.

Ethics and dissemination: This trial has received human ethics approval from the Human Research Ethics Committee (HREC) of RMIT University (Project number 15/12). The study findings will be published in peerreviewed journals and presented at the national and international conferences.

Trial registration: Australia and New Zealand Clinical Trials Register (ANZCTR): ACTRN12612001181897. TGA

\section{Strengths and limitations of this study}

- Atopic dermatitis mainly affects children and this clinical trial was designed to suit the paediatric population. The trial requires verbal consent from the child participants on top of the written consent of their legal guardians. Furthermore, the trial intervention is in the form of a small capsule that would be easy for children to swallow. As an extra safety measure, the trial includes a 'capsule swallow test' to ensure that only children who have no trouble swallowing capsules are included in the trial. The trial also offers an optional 'capsule-swallowing training programme' to potential participants, who will be able to participate in the study should they become successful in swallowing capsules after the training.

- Clinical studies involving Chinese herbal medicine follow the reverse pharmacology method, where clinical studies are conducted prior to pharmacological studies. RMIT Chinese Medicine-106 (RCM-106) was formulated based on the theories, historical and empirical evidence of traditional Chinese medicine. This means that there is a lack of understanding of the pharmacological actions of the formula. Further studies on the formula would be necessary to provide a more complete understanding of the treatment.

- Size of the study sample limits the power of observations.

CTN Scheme: Trial number 2012/0713; Protocol number $15 / 12$.

\section{INTRODUCTION}

Atopic dermatitis (AD) is a chronic, inflammatory skin rash which affects approximately $15-30 \%$ of children and $2-10 \%$ of adults. ${ }^{1}$ The presentation of $\mathrm{AD}$ can vary but 
common symptoms include severe itching, redness and dryness of the skin, weeping or scaring and lichenification. ${ }^{23}$ While rarely fatal, the itch-scratch cycle can lead to disfiguration, sleep disturbances and subsequent lack of self-confidence and low work productivity. ${ }^{45}$ Patients and families are further burdened by the economic costs for disease managements. ${ }^{4}$ The estimated annual expenditure on $\mathrm{AD}$ in the UK was $£ 465$ million $^{6}$; the national direct costs in the USA ranged between US $\$ 364$ million and US $\$ 3.8$ billion. ${ }^{7}$ In Australia, the yearly personal costs of $\mathrm{AD}$ is said to be greater than that of asthma, ranging from $\$ \mathrm{~A} 330$ to $\$ \mathrm{~A} 1255^{8}$. $^{\mathrm{C}}$

The main treatment is to recognise and remove triggering factors, maintain skin hydration and reduce itching and inflammation. ${ }^{3}$ Medication and other forms of management are targeted at symptomatic relief. ${ }^{5}$ The mainstay therapies include topical corticosteroids, topical calcineurin inhibitors and emollient therapy. ${ }^{9-11}$ However, extended use of topical corticosteroids can lead to local and systemic adverse events such as skin atrophy and primarily hypothalamic-pituitary-adrenal axis suppression ${ }^{10}$; topical calcineurin inhibitors causes skin irritation and there are possible malignancy risks with their long-term usage. ${ }^{9}$ The use of emollients is targeted at skin hydration to relieve pruritus and repair skin barrier function, though evidence is lacking. ${ }^{11}$ Patients with $\mathrm{AD}$ often respond poorly or become recalcitrant towards these treatments. ${ }^{12}$

Traditional Chinese medicine (TCM) has been used to treat various conditions, including dermatological conditions. ${ }^{13}$ TCM treatment is expected to have beneficial prospects as it has its own form of diagnosis to enable targeted treatments. According to Yao, ${ }^{14}$ the TCM treatment of AD via syndrome differentiation can regulate the allergy or atopy-prone constitution and has shown promising effects in relieving signs and symptoms, preventing recurrence, maintaining remission and improving quality of life. The recent studies have shown that Chinese herbs have various pharmacological actions, including anti-inflammatory, antibacterial, antifungal and immunosuppressive functions. ${ }^{13}$ Several clinical studies have also shown the potential of TCM treatment of AD. ${ }^{15-17}$ However, systematic reviews have deemed the overall studies to be of 'poor quality', therefore resulting in insufficient evidence for valid conclusions. ${ }^{5} 1819$ Furthermore, there are concerns regarding the safety of Chinese herbal treatment due to reports of serious adverse events such as contact dermatitis, arsenic or mercury poisoning, liver toxicity and other diseases with the use of Chinese herbal medicine. ${ }^{20}$ Armstrong and Ernst ${ }^{18}$ suggested that more studies should be carried out to establish its efficacy, safety, costeffectiveness and mechanism of action.

\section{OBJECTIVE}

In order to address the lack of evidence in the efficacy and safety of TCM treatments of $\mathrm{AD}$, we have conducted systematic reviews on the Chinese herbal medicine treatment of $\mathrm{AD} .{ }^{19}$ The results of the systematic reviews assisted in the rigorous design of this randomised controlled trial (RCT) and in the formulation of the new herbal formula, RMIT Chinese Medicine-106 (RCM-106), for AD. This trial will evaluate the efficacy and safety of RCM-106 in the management of $\mathrm{AD}$ in children in a randomised, doubleblind, placebo-controlled trial. Besides the efficacy and safety of RCM-106, this trial will also evaluate the effects of RCM-106 in improving the quality of life of children affected by AD. Comments and advice were sought from a dermatology expert panel in China to assist in the final formulation of RCM-106. To achieve the trial objective, the following research questions are to be answered: (1) Can RCM-106 reduce the severity of AD in terms of Scoring Atopic Dermatitis (SCORAD) and Patient-Oriented Scoring Atopic Dermatitis (PO-SCORAD) index? (2) Can RCM-106 improve the quality of life of patients when evaluated by the Children's Dermatology Life Quality Index (CDLQI)? (3) Can treatment with RCM-106 reduce the use of other topical remedies for AD? (4) What adverse events does RCM-106 produce in children with moderateto-severe $\mathrm{AD}$ ?

\section{METHODS}

\section{Design}

This study is a double-blind, randomised, parallel-armed, placebo-controlled, clinical trial comparing RCM-106 and placebo capsules in children with moderate-to-severe $\mathrm{AD}$. The rigorous design and protocol were planned in accordance with the findings of previous systematic reviews, ${ }^{51819}$ in combination with the appropriate use of TCM in clinical practice. The study complies with the principles of the Declaration of Helsinki and Good Clinical Practice guidelines. Its reporting will be guided by the CONSORT statement ${ }^{21} 22$ and the relevant extensions related to herbal medicine interventions. ${ }^{23} 24$

The trial will be conducted in RMIT University (City and Bundoora Campuses), Melbourne, Australia. A total of 90 participants will be recruited. After acquiring consent from the participants and their parents/legal guardian, the participants will be enrolled for a trial period of 14 weeks and will be required to attend a total of six visits during the trial. The study consists of a 2-week run-in period, an 8-week treatment period and a 4-week follow-up period. The duration for the run-in, treatment and follow-up was decided based on our previous systematic reviews. ${ }^{19}$ The outline of trial procedures are illustrated in figure 1.

Potential participants will undergo preliminary screening for eligibility during visit 1 by investigators, which will include a registered medical practitioner. Eligible participants will then undergo initial assessments for baseline data collection, which include the SCORAD, PO-SCORAD, CDLQI, a Chinese Medicine Questionnaire, measurement of vital signs (temperature, blood pressure and heart rate), full blood count, total IgE, eosinophil count, kidney function test and liver function test. A daily 


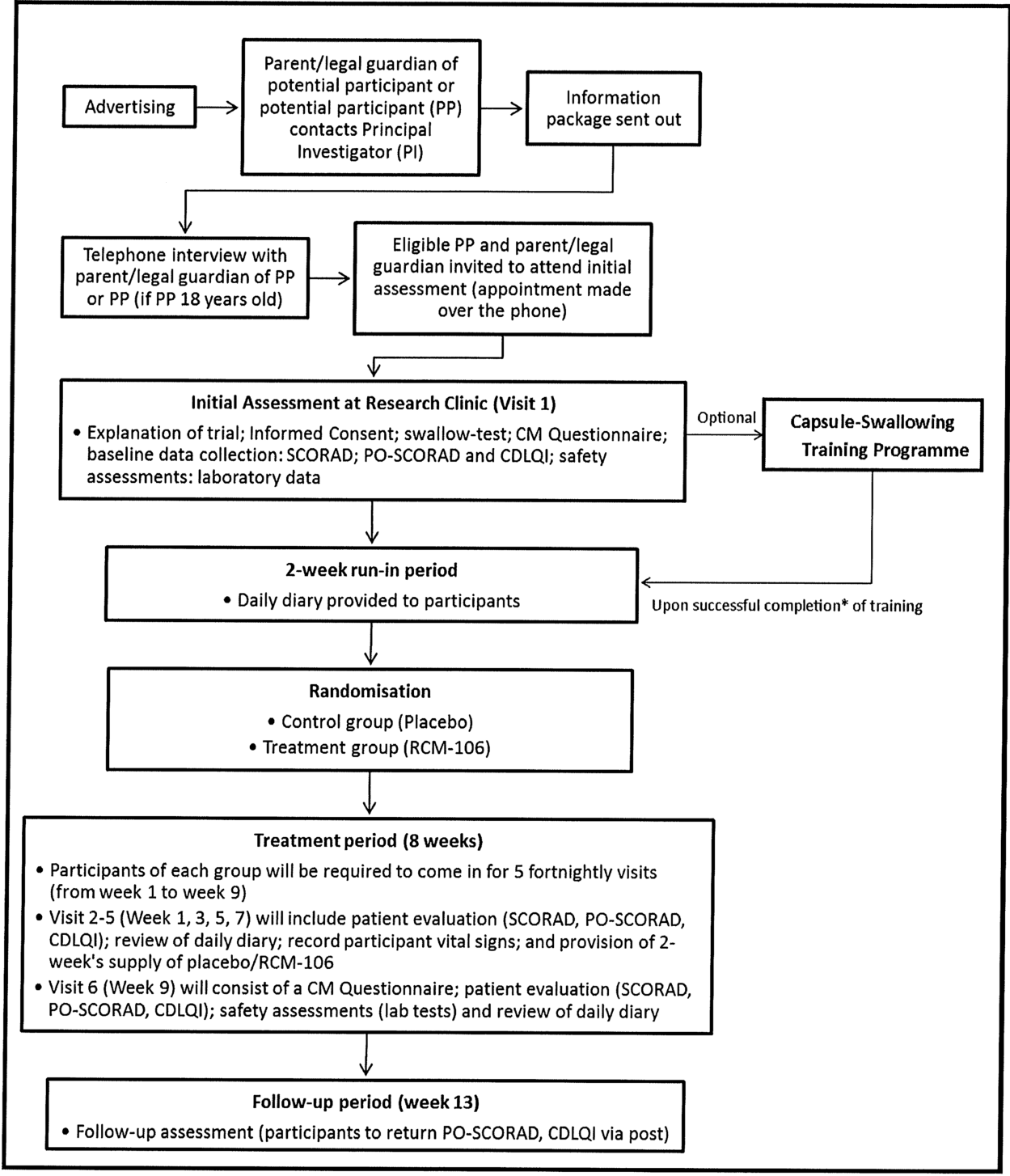

*Successful completion = able to swallow size \#1 capsules

Figure 1 Outline of trial procedure.

diary will then be given to record the occurrence of adverse events and use of topical treatments during the 2-week run-in period. After the run-in period, participants will be randomly assigned to either the treatment (RCM-106) group or the control (placebo) group and the treatment period will start. During the fortnightly clinic visits, participants will be given 2 weeks' worth of RCM-106 or placebo capsules and daily diaries for the fortnight. Vital signs, SCORAD, PO-SCORAD and CDLQI will be assessed as well. During the treatment period, participants will also be required to record their medication intake, including trial interventions and occurrence of adverse events to assist with compliance monitoring and acceptability of intervention. After the treatment period, participants will be given the PO-SCORAD, CDLQI and a prepaid envelope and be asked to complete and return the outcome assessment instruments at the end of the 4-week follow-up period.

\section{Participants}

Participants of the trial will be recruited via advertising on the Internet (AD/eczema online communities; association websites; Facebook/Twitter; RMIT website). Advertising in the form of posters or flyers will be made available in RMIT University (Bundoora and City campuses), children's hospital or paediatric wards of hospitals, medical centres, clinics of dermatologists and Chinese medicine practitioners, primary and secondary schools and community libraries of surrounding suburbs in Melbourne. Advertising via other forms of media (radio, newspaper and television) may be used if necessary. Interested participants or their parents/legal guardian can make enquiries via email or telephone. Participant information and consent forms (for parents/legal guardian) will be sent out to potential participants prior to the telephone interview and scheduling of the first visit. 


\section{Inclusion criteria}

The trial will include participants of both genders who are aged 6-18 years, diagnosed with $\mathrm{AD}$ according to the UK Working Party's Diagnostic Criteria (UK Diagnostic Criteria) ${ }^{25}$ and of a moderate-to-severe severity (defined as $\mathrm{SCORAD} \geq 25) .{ }^{26}$ All participants have to agree to abstain from alcohol during the period of the trial and not be involved in other trials. Written consent will be sought from the parents/legal guardian of the participants, as well as from the participants who are able to read and write fluently. Verbal assent will be sought from all other participants. In addition, all potential participants will be required to undergo a 'swallow-test' to prove their ability to swallow size \#1 capsules (approximately $19.4 \mathrm{~mm}$ in length and $6.91 \mathrm{~mm}$ in diameter). The participants who are unable to pass the test can opt to undergo a 'capsule-swallowing training programme' and participate in the trial on successful completion of the programme (ie, able to swallow size \#1 capsules).

\section{Exclusion criteria}

The participants are not eligible to participate in the trial if (1) there is overt bacterial infection or concurrent systemic disease (except asthma and allergic rhinitis); (2) they are pregnant, breastfeeding, intending to get pregnant or are women of childbearing age refusing contraception; (3) they are unable to swallow size \#1 capsules and refuse to participate in the 'capsule-swallowing training programme'; (4) they have a history of sensitivity towards Chinese herbal medicine; (5) they have an abnormal full blood count (with the exception of parameters related to $\mathrm{AD}$, such as eosinophil count and total IgE), renal or liver function tests; (6) they have been using Chinese herbs, systemic steroids, antibiotics, phototherapy or other immunemodulating drugs 4 weeks prior to the study; (7) they are unable to refrain from using other therapies during the trial period (except for the use of topical, non-Chinese medicine therapies when necessary); or (8) they are unable to understand English.

\section{Ethics and trial registration}

Any amendments to the study protocol will be submitted to the Human Research Ethics Committee (HREC) for approval. The trial has also been registered with the Australia and New Zealand Clinical Trials Register (ANZCTR; ACTRN12612001181897) and the Clinical Trial Notification (CTN) Scheme with the Therapeutic Goods Administration (TGA; trial number 2012/0713).

\section{Swallow-test}

As the trial intervention will be herbal extracts or placebo encapsulated in size \#1 capsules, we have introduced a 'swallow-test' as a safety precaution. The swallow-test is to ensure that the participants are capable of swallowing capsules of that size and therefore eligible to participate in the study. The swallow-test will require potential participants to swallow an empty, size \#1, vegetarian capsule. Water will be supplied during the test and the participants will not be allowed to use other drinks to complete the test. The participants who are unable to swallow the capsule will be given the option of undertaking the "capsule-swallowing training programme' or be excluded from the study.

\section{Capsule-swallowing training programme}

Potential participants who suit the inclusion criteria but are unable to swallow a size \#1 capsule (either selfreported or after the swallow test) may choose to undergo a 'capsule-swallowing training programme', if they are still keen to participate in the study. Studies have shown that various forms of training programmes have been successful in teaching children to swallow pills. ${ }^{27-29}$ Parents of the participants will be given training guidelines (figure 2) and a supply of empty capsules of various sizes for the training. The guidelines for the 'capsule-swallowing training programme' were modified from the 'Teach children how to swallow tablets and capsules' guidelines by the Royal Children's Hospital. ${ }^{30}$ Should the participants succeed in swallowing size \#1 capsules after the training, they will be eligible to participate in the study.

\section{Randomisation and blinding}

Randomisation will be carried out after the run-in period using block randomised sequences generated by computer. Each participant will be assigned an ID code. An independent statistician will be responsible for the stratified randomisation to ensure the balance in gender and disease severity of both groups. According to the SCORAD classification of severity, an SCORAD index of 25-50 is considered moderate $\mathrm{AD}$ and an index above 50 is considered severe $\mathrm{AD}{ }^{26}$

Blinding will be carried out using treatment codes and prepacking of placebo and RCM-106 capsules, which are identical in appearance, taste and scent. The codes and labelling will be recorded in a password protected computer programme. Participants, investigators and outcome assessors will remain blinded to the treatment allocation until after the study has been completed.

\section{Sample size}

The sample size calculation for this study was based on the effect size calculations from the severity scores of two studies-one study from Taiwan using a similar Chinese herbal formula for $\mathrm{AD}^{17}$ and another study comparing prebiotic and symbiotic treatment. ${ }^{31}$ With the effect size estimate of 1.76 calculated from the Taiwan study, ${ }^{17}$ to achieve $95 \%$ power with a significance level of 5\%, 10 participants per group would be required. On the basis of end SCORAD in Wu et al $\mathrm{s}^{31}$ study, an effect size estimate of 0.64 was calculated. To achieve $85 \%$ power with a significance level of $5 \%$ with the effect size estimate of $0.64,45$ participants per group would be required. Considering that the former study had some methodological differences to this study and used a much larger treatment dose, while the latter used 


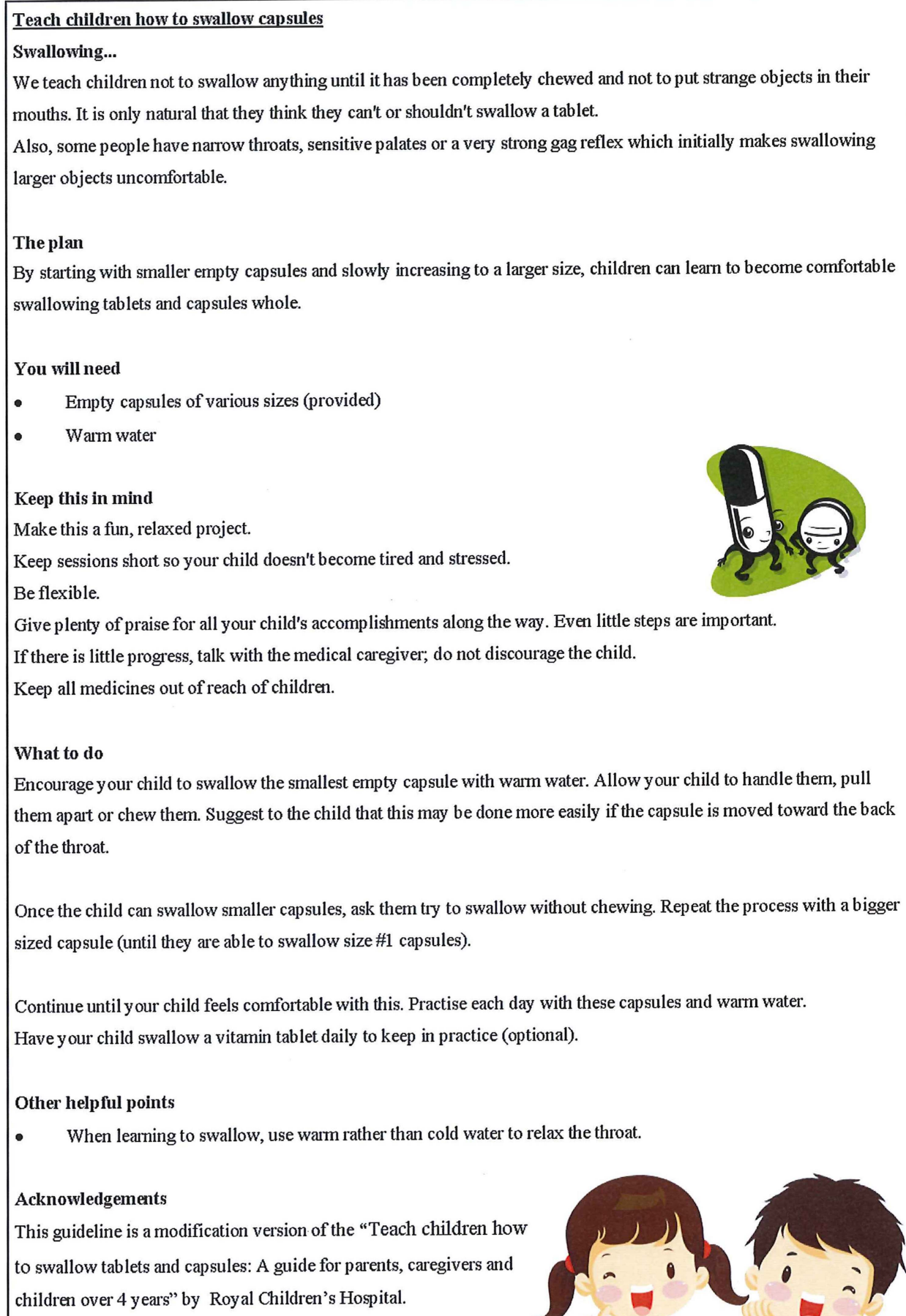

Give plenty of praise for all your child's accomplishments along the way. Even little steps are important.

If there is little progress, talk with the medical caregiver; do not discourage the child.

Keep all medicines out of reach of children.

What to do

Encourage your child to swallow the smallest empty capsule with warm water. Allow your child to handle them, pull them apart or chew them. Suggest to the child that this may be done more easily if the capsule is moved toward the back of the throat.

Once the child can swallow smaller capsules, ask them try to swallow without chewing. Repeat the process with a bigger sized capsule (until they are able to swallow size \#1 capsules).

Continue until your child feels comfortable with this. Practise each day with these capsules and warm water.

Have your child swallow a vitamin tablet daily to keep in practice (optional).

Other helpful points

- When learning to swallow, use warm rather than cold water to relax the throat.

\section{Acknowledgements}

This guideline is a modification version of the "Teach children how

to swallow tablets and capsules: A guide for parents, caregivers and

children over 4 years" by Royal Children's Hospital.

Figure 2 Capsule swallowing training guidelines-modified from the 'Teach children how to swallow tablets and capsules' guidelines by the Royal Children's Hospital (http://www.rch.org.au/pharmacy/clinical_services/Teach_children_how_to_swallow_ tablets_and_capsules).

a similar methodology but different form of intervention, a sample size of 90 participants, inclusive of $20 \%$ dropout compensation, will be applied for this study.

\section{Trial interventions}

The treatment interventions are RCM-106 herbal extract capsules and matching placebo capsules, which are identical in appearance, taste and odour. The RCM-106 was formulated based on the classic formula, Xiao Feng San. Each capsule will consist of the herbal granule extracts of seven plant herbal substances (Fang Feng $75 \mathrm{mg}$, Chao Bai Zhu $75 \mathrm{mg}$, Ku Shen $75 \mathrm{mg}$, Sheng Di Huang $100 \mathrm{mg}$, Bai Shao $50 \mathrm{mg}$, Gan Cao $50 \mathrm{mg}$ and Bai Xian Pi $75 \mathrm{mg}$ ), all of which are listed as approved 
substances for human consumption by the TGA. The dosage of each raw herbal ingredient is within the dose range as recorded in the Pharmacopoeia of the People's Republic of China. ${ }^{32}$ The placebo capsules will consist of herbal starch which contains no active ingredients.

The capsules will be produced by a manufacturer that holds a TGA approved Good Manufacturing Practice (GMP) certificate. The herbal extracts will be of a concentration ratio of $7: 1$. The capsules will be dispensed as $500 \mathrm{mg}$, size \#1, vegetarian capsules for oral intake and packed in sealed bottles containing 2-week's dose of capsules. Participants aged 6-11 years will be required to take three capsules while the participants aged 12-18 years will be required to take six capsules, at each dosing period, twice daily, for the treatment period of 8 weeks. The dosage of RCM-106 for children was determined by taking into consideration the age-to-dose guidelines published by the Nanjing College of Traditional Chinese Medicine ${ }^{33}$ and the conversion table of Von Harnack. ${ }^{34}$ Quality control checks on the packaging and contents of the treatment interventions, including checks for potential contaminants such as heavy metal or steroids, will be undertaken by the manufacturers to ensure their stability and quality.

The placebo capsules will consist of herbal starch, which is starch made from the herbal dregs of the active intervention after extraction and therefore have a similar appearance, smell and taste to RCM-106 extracts but contain no active constituents. ${ }^{35}$

Throughout the treatment period, participants are not permitted to use any systemic treatments, including supplements and complementary medicines. The use of other topical, non-Chinese medicine, such as topical corticosteroids, is not encouraged but is allowed to be used on an 'as needed' basis. Participants will be given a daily diary to record their trial medication compliance as well as usage of any other therapies and occurrence of adverse events. Participants will be asked to return their medication bottles to enable the counting of left-over capsules as well as part of participant adherence monitoring.

Chinese medicine diagnosis and syndrome differentiation Participants will be asked to complete a Chinese Medicine Questionnaire at the time of recruitment and at the end of the treatment period. This questionnaire is developed based on the State Administration of TCM's Criteria of Diagnosis and Therapeutic Effect of Disease and Syndromes in TCM. ${ }^{36}$ The questionnaire is used to assist the TCM diagnosis and syndrome differentiation of AD. While RCM-106 does not target a specific syndrome, this questionnaire will allow comparison of treatment effects of different TCM syndromes. This information is invaluable as it may help bridge the gap between TCM and Western medicine when coupled with pharmacological studies of the formula.

\section{Outcomes measures}

The primary outcome measures include the evaluation of disease severity using two validated instruments:

\section{- SCORAD}

\section{- PO-SCORAD}

Secondary outcome measures include the evaluation of quality of life using the validated CDLQI; occurrence of adverse events and usage of other therapies as recorded in the daily diary and safety components which include full blood count, eosinophil count, total $\operatorname{IgE}$ and liver and kidney function tests.

Participants will be required to complete the SCORAD, PO-SCORAD and CDLQI at each visit, six times in total (during initial assessment, weeks 1, 3, 5, 7 and 9). The PO-SCORAD and CDLQI will also need to be completed and returned via post at the end of the follow-up period. Participants will also be required to complete their daily diaries from the start of the run-in period until the end of the treatment period. Blood tests for full blood count, eosinophil count, total $\mathrm{IgE}$ and liver and kidney function tests will be conducted at initial assessment and during the final clinic visit (week 9).

All adverse events will be followed up from the date it is brought to the investigator's attention until the adverse event has been resolved. In the occasion of a severe adverse event, the event will be recorded and immediately reported to the RMIT HREC, followed by a detailed written report. Participants will be identified by their ID codes to maintain confidentiality.

Participants may be withdrawn from the trial by the investigator if a serious adverse event occurs. Participants are also permitted to withdraw at will at any time of the trial. All withdrawn cases who have received the intervention will be contacted 4 weeks following withdrawal, as the follow-up period, to obtain information with regard to their condition using PO-SCORAD and CDLQI.

\section{Statistical analysis}

The trial data will be processed and analysed by an independent statistician, who will be blinded to participant allocation, under the supervision of the School of Mathematical and Geospatial Sciences at RMIT University. Intention-to-treat analysis will be applied to include all randomised participants. Data will be summarised as means and SDs and analysed using the Statistical Package for the Social Sciences (SPSS, Windows V.19.0). The statistical procedure to be employed is repeated measures analysis of variance utilising the General Linear Model (GLM). Data from non-repeated measures will be analysed by $\mathrm{t}$ tests. Outcome measures with categorical responses will be analysed using $\chi^{2}$ and Fisher exact tests. All $\mathrm{p}$ values will be two-tailed and at $\alpha=0.05$. To assist safety monitoring, interim analysis will be conducted.

\section{DISCUSSION}

This study protocol was designed to suit the paediatric population. To facilitate blinding and ease of drug 
dosing and delivery, we have chosen to administer RCM-106 as small, size \#1 capsules. To reduce the risk of choking hazards, the ability to swallow capsules has been included in our participant criteria. On advice from a paediatric allergist from the Royal Children's Hospital, as a precaution against false reports of the ability to swallow capsules by eager participants or their parents/ legal guardian, this protocol includes a 'swallow-test' during screening to ensure that the included participants are indeed able to swallow capsules without difficulty. The protocol also offers an optional 'capsuleswallowing training programme' for potential participants who are unable to swallow capsules on screening. Anecdotal evidence suggests that children aged 6 years or younger would be able to take solid dosage forms with adequate support and training ${ }^{37}$; furthermore, studies on various methods of pill-swallowing training have been shown to be successful. ${ }^{27-29}$

The previous systematic reviews on Chinese herbal medicine for $\mathrm{AD}$ concluded that the quality of trials were poor, and therefore did not allow for valid conclusions. The systematic reviews highlighted several methodological and reporting aspects to be improved and these have been incorporated in this clinical trial. These include, but are not limited to the use of validated diagnostic criteria and outcome measure instruments; the use of capsules to improve compliance; and dosage determination based on the Pharmacopoeia of the People's Republic of China. ${ }^{32}$ Furthermore, the reporting of the trial will abide by the relevant CONSORT guidelines.

The primary outcome measures include SCORAD and PO-SCORAD which are validated instruments to assess disease severity and extent through a scoring system. A higher score represents a more severe condition. SCORAD is the assessment by a third-party assessor, whereas PO-SCORAD is the assessment by the patient. $^{38}{ }^{39}$ SCORAD and PO-SCORAD scores corelate well to each other. ${ }^{40}$ The SCORAD and PO-SCORAD will assist in the evaluation of the efficacy of RCM-106.

The secondary outcome measures include the evaluation of quality of life using CDLQI, occurrence of adverse events, total amount of other therapies used and laboratory tests. The CDLQI consists of 10 questions to determine the impact of dermatological conditions on the quality of life of affected children. ${ }^{41}$ Each question can be given a score of $0-3$, with a total score of 30 . A higher score represents a lower quality of life or a higher impact on quality of life. Although the CDLQI was intended for children up to the age of 16 years, ${ }^{41}$ it has shown to be applicable to children up to the age of 21 years. ${ }^{42}$ For this trial, the CDLQI will be used for children up to 18 years. Participants aged $6-11$ years will be given the English cartoon version of the instrument, while the participants aged $12-18$ years will be given the English text version. The CDLQI is used to evaluate whether RCM-106 is able to improve the quality of life of patients with $\mathrm{AD}$.
The daily diary will include records of trial intervention intake, other treatments used and occurrence of adverse events. The record of trial intervention compliance will assist with the evaluation of the acceptability of the intervention and reflect the efficacy of the intervention; the record of other treatments used will provide an insight on whether RCM-106 is able to reduce the requirement for other remedies, and thus suggests its efficacy; the record of adverse events will assist with the assessment of tolerability of RCM-106.

The laboratory tests that would be assessed include full blood count, eosinophil count, total $\mathrm{IgE}$ and liver and kidney function tests. Increased eosinophil count is more highly observed in patients with $\mathrm{AD}$ than in healthy individuals. ${ }^{43}$ Total $\mathrm{IgE}$ is measured as $\mathrm{AD}$ is defined as the IgE-related form of dermatitis by the World Allergy Organisation $^{44}$; and elevated serum IgE is seen in up to $85 \%$ of $\mathrm{AD}$ cases. ${ }^{45}$ The full blood count, liver function test and kidney function test are to assist with safety monitoring and assessment of tolerability of RCM-106.

The results of this RCT will provide clinical data on the efficacy and safety of RCM-106 in reducing the severity of $\mathrm{AD}$ and improving the quality of life of patients with AD. Positive results from the RCT can lead to a better management of $\mathrm{AD}$ to help patients. This RCT will also contribute to the understanding and treatment of $\mathrm{AD}$ from Chinese medicine perspectives.

\section{Author affiliations}

${ }^{1}$ Traditional \& Complementary Medicine Research Program, Health Innovations Research Institute, School of Health Sciences, RMIT University, Bundoora, Victoria, Australia

${ }^{2}$ Guangdong Provincial Academy of Chinese Medical Sciences, Guangzhou, China

${ }^{3}$ School of Mathematical and Geospatial Sciences, RMIT University, Melbourne, Victoria, Australia

Acknowledgements The authors would like to express their gratitude to the dermatology expert panel set up by the Guangdong Provincial Academy of Chinese Medical Sciences as well as Professor Jerry Zhang (RMIT University), who have provided valuable advice with regard to the formulation of RMIT Chinese Medicine-106 (RCM-106). They would also like to thank Professor Marc Cohen (RMIT University) for agreeing to lend his assistance as the registered medical practitioner for participant screening and monitoring during the study; Professor Alan Bensoussan (University of Western Sydney) for his valuable guidance and advice on herbal safety in trials; and paediatric allergist and immunologist from the Royal Children's Hospital, Dr Dean Tey, who provided input on drug delivery in children. The guidelines for the 'capsule-swallowing training program' were modified from the 'Teach children how to swallow tablets and capsules' guidelines by the Royal Children's Hospital.

Contributors HYT, GBL and ALZ designed the study and prepared the ethics application. CCX, CDC and DC reviewed the study design and protocol. CDC is the statistician of the study and provided input on the sample size calculation and data analysis of the study. HYT wrote the protocol manuscript, with critical review and feedback from all other authors. All authors read and approved the final version of the manuscript.

Funding This research received no specific grant from any funding agency in the public, commercial or not-for-profit sectors.

Competing interests HYT received a partial fee waiver scholarship in 2012 and a full fee waiver scholarship in 2013 from the RMIT University for her $\mathrm{PhD}$ studies. 
Ethics approval This trial has received human ethics approval from the Human Research Ethics Committee (HREC) of RMIT University, Australia (Project number 15/12).

Provenance and peer review Not commissioned; externally peer reviewed.

Data sharing statement Further details of the study protocol can be requested from the corresponding author.

Open Access This is an Open Access article distributed in accordance with the Creative Commons Attribution Non Commercial (CC BY-NC 3.0) license, which permits others to distribute, remix, adapt, build upon this work noncommercially, and license their derivative works on different terms, provided the original work is properly cited and the use is non-commercial. See: http:// creativecommons.org/licenses/by-nc/3.0/

\section{REFERENCES}

1. Bieber T. Atopic dermatitis. N Engl J Med 2008;358:1483-94.

2. Chang C, Keen CL, Gershwin ME. Treatment of eczema. Clin Rev Allergy Immunol 2007;33:204-25.

3. Feingold S, Huang C, Kristal L, et al. Eczemas. Curr Probl Dermatol 1998:10:41-90.

4. Hoare C, Li Wan Po A, Williams H. Systematic review of treatments for atopic eczema. Health Technol Assess 2000;4:1-191.

5. Zhang W, Leonard T, Bath-Hextall FJ, et al. WITHDRAWN: Chinese herbal medicine for atopic eczema. Cochrane Database Syst Rev 2013;(9):CD002291.

6. Herd RM, Tidman MJ, Prescott RJ, et al. The cost of atopic eczema Br J Dermatol 1996;135:20-3.

7. Mancini AJ, Kaulback K, Chamlin SL. The socioeconomic impact of atopic dermatitis in the United States: a systematic review. Pediatr Dermatol 2008;25:1-6.

8. Su JC, Kemp AS, Varigos GA, et al. Atopic eczema: its impact on the family and financial cost. Arch Dis Child 1997;76:159-62.

9. Kobayashi $\mathrm{H}$, Ishii M, Takeuchi $\mathrm{S}$, et al. Efficacy and safety of a traditional herbal medicine, hochu-ekki-to in the long-term management of Kikyo (Delicate Constitution) patients with atopic dermatitis: a 6-month, multicenter, double-blind, randomized, placebo-controlled study. Evid Based Complement Alternat Med 2010;7:367-73

10. Buys LM. Treatment options for atopic dermatitis. Am Fam Physician 2007;75:523.

11. Plotz SG, Ring J. What's new in atopic eczema? Expert Opin Emerg Drugs 2010;15:249-67.

12. Hsu C-J, Wang L-F. Emerging treatment of atopic dermatitis. Clin Rev Allergy Immunol 2007;33:199-203.

13. Bedi MK, Shenefelt PD. Herbal therapy in dermatology. Arch Dermatol 2002;138:232-42.

14. Yao $\mathrm{CH}$. Experience in treating atopic dermatitis depending on syndrome differentiation of traditional Chinese medicine. Zhongguo Zhong xi yi jie he za zhi Zhongguo Zhongxiyi jiehe zazhi = Chinese journal of integrated traditional and Western medicine/Zhongguo. Zhen Ci Yan Jiu 2008;28:682.

15. Sheehan MP, Atherton DJ. A controlled trial of traditional Chinese medicinal plants in widespread non-exudative atopic eczema. $\mathrm{Br} J$ Dermatol 1992;126:179-84.

16. Sheehan MP, Rustin MHA, Atherton DJ, et al. Efficacy of traditional Chinese herbal therapy in adult atopic dermatitis. Lancet 1992;340:13-17.

17. Cheng HM, Chiang LC, Jan YM, et al. The efficacy and safety of a Chinese herbal product (Xiao-Feng-San) for the treatment of refractory atopic dermatitis: a randomized, double-blind, placebo-controlled trial. Int Arch Allergy Immunol 2011;155:141-8.

18. Armstrong NC, Ernst E. The treatment of eczema with Chinese herbs: a systematic review of randomized clinical trials. $\mathrm{Br} \mathrm{J} \mathrm{Clin}$ Pharmacol 1999;48:262-4.

19. Tan HY, Zhang AL, Chen D, et al. Chinese herbal medicine for atopic dermatitis: a systematic review. J Am Acad Dermatol 2013;69:295-304.

20. Boneberger S, Rupec RA, Ruzicka T. Complementary therapy for atopic dermatitis and other allergic skin diseases: facts and controversies. Clin Dermatol 2010;28:57-61.

21. Moher D, Hopewell S, Schulz KF, et al. CONSORT 2010 explanation and elaboration: updated guidelines for reporting paralle group randomised trials. Int J Surg 2012;10:28-55

22. Schulz KF, Altman DG, Moher D. CONSORT 2010 statement: updated guidelines for reporting parallel group randomized trials. Open Med 2010;4:E60-8.
23. Gagnier JJ, Boon $\mathrm{H}$, Rochon $\mathrm{P}$, et al. Recommendations for reporting randomized controlled trials of herbal interventions: explanation and elaboration. J Clin Epidemiol 2006;59:1134-49.

24. Gagnier JJ, Boon $\mathrm{H}$, Rochon $\mathrm{P}$, et al. Reporting randomized, controlled trials of herbal interventions: an elaborated CONSORT statement. Ann Intern Med 2006;144:364-7.

25. Williams HC, Burney PGJ, Hay RJ, et al. The UK working party diagnostic-criteria for atopic-dermatitis. 1 Derivation of a minimum set of discriminators for atopic-dermatitis. $\mathrm{Br} J$ Dermatol 1994;131:383-96

26. Oranje AP, Glazenburg EJ, Wolkerstorfer A, et al. Practical issues on interpretation of scoring atopic dermatitis: the SCORAD index, objective SCORAD and the three-item severity score. Br. J. Dermatol 2007;157:645-48.

27. Beck $\mathrm{MH}$, Cataldo $\mathrm{M}$, Slifer $\mathrm{KJ}$, et al. Teaching children with attention deficit hyperactivity disorder (ADHD) and autistic disorder (AD) how to swallow pills. Clin Pediatr (Phila) 2005;44:515-26.

28. Garvie PA, Lensing S, Rai SN. Efficacy of a pill-swallowing training intervention to improve antiretroviral medication adherence in pediatric patients with HIV/AIDS. Pediatrics 2007;119:e893-9.

29. Kaplan BJ, Steiger RA, Pope J, et al. Successful treatment of pill-swallowing difficulties with head posture practice. Paediatr Child Health 2010;15:e1-5.

30. The Royal Children's Hospital Melbourne. Teach children how to swallow tablets and capsules: a guide for parents, caregivers and children over 4 years. Secondary Teach children how to swallow tablets and capsules: a guide for parents, caregivers and children over 4 years 14 March 2013. http://www.rch.org.au/pharmacy/ clinical_services/Teach_children_how_to_swallow_tablets_and_ capsules/

31. Wu KG, Li TH, Peng HJ. Lactobacillus salivarius plus fructo-oligosaccharide is superior to fructo-oligosaccharide alone for treating children with moderate to severe atopic dermatitis: a double-blind, randomized, clinical trial of efficacy and safety. $\mathrm{Br} J$ Dermatol 2012;166:129-36

32. The State Pharmacopoeia Commission of the People's Republic of China. Pharmacopoeia of the People's Republic of China. Beijing: Chemical Industry Press, 2000.

33. Chen JK, Chen TK. Chinese herbal formulas and applications: pharmacological effects. Calif: Art of Medicine Press, 2009.

34. Ministry of Health, Labour and Welfare. Yakuzai-shi Kokka Shiken Shutsudai Kijun (the Criteria for the National Examination for Pharmacists). Tokyo: Ministry of Health, Labour and Welfare of Japan, 2010.

35. Lenon GB, Li KX, Chang YH, et al. Efficacy and safety of a Chinese herbal medicine formula (RCM-104) in the management of simple obesity: a randomized, placebo-controlled clinical trial. Evid Based Complement Alternat Med 2012;2012:435702.

36. The State Administration of Traditional Chinese Medicine of the People's Republic of China. Criteria of diagnosis and therapeutic effect of diseases and syndromes in traditional Chinese medicine. Beijing, China: Nanjing University Press, 1994.

37. European Medicines Agency. Reflection paper: formulations of choice for the paediatric population. 2006.

38. [No authors listed]. Severity scoring of atopic dermatitis: the SCORAD index. Consensus Report of the European Task Force on Atopic Dermatitis. Dermatology 1993;186:23-31.

39. Vourc'h-Jourdain M, Barbarot S, Taieb A, et al. Patient-oriented SCORAD: a self-assessment score in atopic dermatitis-a preliminary feasibility study. Dermatology 2009;218:246-51.

40. Stalder JF, Barbarot S, Wollenberg A, et al. Patient-Oriented SCORAD (PO-SCORAD): a new self-assessment scale in atopic dermatitis validated in Europe. Allergy 2011;66:1114-21.

41. Lewis-Jones MS, Finlay AY. The Children's Dermatology Life Quality Index (CDLQI): initial validation and practical use. $\mathrm{Br} J$ Dermatol 1995;132:942-9.

42. Hon KLE, Leung TF, Ng PC, et al. Efficacy and tolerability of a Chinese herbal medicine concoction for treatment of atopic dermatitis: a randomized, double-blind, placebo-controlled study. Br J Dermatol 2007;157:357-63.

43. Schmid P, Simon D, Simon HU, et al. Epidemiology, clinical features, and immunology of the "intrinsic" (non-IgE-mediated) type of atopic dermatitis (constitutional dermatitis). Allergy 2001;56:841-9.

44. Möhrenschlager M, Darsow U, Schnopp C, et al. Atopic eczema: what's new? J Eur Acad Dermatol Venereol 2006;20:503-13.

45. Leung DYM. Atopic dermatitis: immunobiology and treatment with immune modulators. Clin Exp Immunol 1997;107(Suppl 1):25-30. 